\title{
Tracking for proton Computed Tomography
}

\section{Carlo Civinini ${ }^{1}$}

INFN-Firenze

Via G. Sansone, 1-50019 Sesto Fiorentino (FI), Italy

E-mail: Carlo.Civininiefi.infn.it

In hadron therapy a highly conformed irradiation field is delivered to the target by precisely moving the particle beam and, at the same time, modulating its energy with the aim to cover the tumor volume with the requested dose sparing the surrounding healthy tissues as much as possible. To setup a robust treatment plan the Stopping Power (SP) map for each patient should be measured and the volume to be irradiated precisely located.

To improve the precision of the SP map determination the use of a proton beam to perform a 'proton Computed Tomography' (pCT) could be the ideal solution. To be effective in reducing the uncertainties in dose spatial distribution, this novel method should keep the SP map spatial resolution below one millimeter.

Tracking in presence of large multiple scattering will be discussed in these proceedings together with the concept of 'most likely path' (MLP). A generalization of the studies on this issue will be introduced with the aim to describe instrumental effects on the MLP spatial resolution.

The pCT apparatus, based on a Silicon microstrip tracker followed by a calorimeter to measure single protons trajectory and their residual energy, will be reviewed with particular emphasis on the R\&D ongoing on this subject and the results already obtained. New prototypes presently under construction, which will be suitable for pre-clinical studies, will be described too.

22nd International Workshop on Vertex Detectors (Vertex 2013)

September 15-20, 2013

Lake Starnberg, Germany

\footnotetext{
${ }^{1}$ Speaker
} 


\section{Introduction}

The use of proton beams for cancer treatment was proposed by R. Wilson in 1946 [1]. In his seminal paper, written in view of the forthcoming operation of high energy accelerators, the author discussed the characteristics of the interactions of hadrons with biological tissues starting with the most important one: the end of range increase of the specific energy loss discovered by W.H. Bragg about 40 years before [2]. This property makes the use of hadrons of a paramount importance for tumors located very close to critical anatomical structures. The technological path of this therapy has led to a number of treated patients of more than 100000 , most of them concentrated in the last two decades [3].

In a hadron therapy session the tumor volume is irradiated pixel by pixel moving the particle beam and, at the same time, modulating its energy to correctly place the Bragg's peak in the selected point. The target transverse position with respect to the beam, once the patient has been correctly positioned, is determined by an X-ray Computed Tomography (X-CT), while the desired target depth is reached adjusting the beam energy accordingly to the path integral of the proton stopping power (SP) distribution from the entry point to the destination. Presently the SP maps are extracted from X-CT images converting the photon attenuation coefficients (or Hounsfield units) into SP using appropriate calibrations [4]. This method introduces uncertainties which, translated into the position of the Bragg peaks, can be as large as $3 \mathrm{~mm}$ [5].

A second source of error comes from the patient positioning. In fact the tumor location is done by an X-CT taken in a set-up which is, in general, different from the irradiation one.

These sources of error could be mitigated by a 'proton Computed Tomography' (pCT) system. With such an apparatus the SP maps could be directly extracted using the same irradiation beam but with a much reduced intensity and an increased energy to allow protons to cross the patient's body carrying information on the traversed tissues. The positioning problem could be tackled too since the pCT set-up would be the same of the irradiation one with the patient aligned and treated in one go.

\section{The proton Computed Tomography}

\subsection{Working principles}

A pCT apparatus is made by a tracking section, to reconstruct the proton trajectory up- and down-stream the object under study (phantom), followed by a calorimeter to measure the proton residual energy and to trigger the data acquisition system (DAQ). As detailed in Sect. 3, to reduce the effect of Multiple Coulomb Scattering (MCS) the apparatus' DAQ should be able to record information event-by-event with the aim to track the path of each single proton in the phantom. Data is taken with the phantom rotated at different angles and tomographic reconstruction techniques are used to reconstruct the 3D SP maps [6]. To be competitive with the present SP measurements the pCT reconstructed maps should have position resolution better than one millimetre and electron density resolution better than 1\% [7]. Finally, to keep the time needed to acquire images within few minutes, the DAQ should be run at a frequency of one $\mathrm{MHz}$ or more. 


\subsection{Historical development}

A.M. Koehler, exploiting the abrupt beam flux change for thicknesses near to the particle range, firstly uses protons to obtain high contrast radiographies [8]. Following a proposal to check in vivo the proton ranges for treatment planning verification [9] a München University and Paul Sherrer Institute group realized, in the late '90s, a proton radiography system [10].

The pCT approach based on the measurement of the trajectory of a single particle and its residual energy, has been proposed by University of California Santa Cruz (UCSC) and Loma Linda University Medical Center (LLUMC) groups in 2003 [11]. This apparatus includes a silicon microstrip tracker to measure the proton trajectory and, using the specific energy loss method, its energy. A crystal calorimeter has been subsequently added [12] which was replaced by a plastic scintillator range counter [13] in an extended field of view prototype.

Another system, which make use of a silicon microstrip tracker and a YAG:Ce calorimeter, has been realized [14-16] by an INFN collaboration which is presently working on an extended area device for pre-clinical studies [17]. Recently a Japanese collaboration has developed a pCT system again based on silicon tracker and a NaI(Tl) calorimeter [18].

The apparatus proposed by TERA (Hadron therapy Oncological Foundation), includes two planes of GEM (Gas Electron Multiplier) detectors and a scintillator stack [19]. A system, again based on measuring the path and residual range, has been constructed by the PSI group using an Emulsion Cloud Chamber (ECC) made of nuclear emulsions interleaved with tissue-equivalent plastic plates [20].

This paper describes the tracking part of apparatus which use silicon microstrip detectors.

\section{The 'Most Likely Path' determination}

The MCS is the main difficulty to meet the required spatial resolution in pCT. In fact targets could be as thick as $20 \mathrm{~cm}$ water equivalent: in this case a $200 \mathrm{MeV}$ proton undergoes to an rms MCS angle of $40 \mathrm{mrad}$ which corresponds to an rms of the projected displacement, onto a surface placed at the very exit of the phantom, of $3.2 \mathrm{~mm}$. With these values simple proton radiographies cannot be used to extract the SP map with a sub-millimetre precision.

A possible solution to this problem is to use the full information on a single event basis: particle entry and exit points and directions are used as input to an algorithm which evaluates the Most Probable Path (MLP) of each proton inside the phantom [21]. Using this method the maximum value of the error envelope on a transverse coordinate of the MLP of a $200 \mathrm{MeV}$ energy proton in $20 \mathrm{~cm}$ of water could be kept well below one millimeter [21].

\subsection{MLP generalization}

Starting from the compact MLP formula reported in [22] an extension of its field of validity has been worked out to take into account:

- real geometry of a pCT apparatus where, to make room for rotating the phantom, a fraction of the proton trajectory is necessarily outside the material;

- instrumental effects of the tracker detectors.

The instrumental effects are the error on the measured trajectory positions and directions at the tracking planes. Because of the large strip pitch used in the pCT silicon microstrip trackers, 
a realistic estimation of the position resolution could be the pitch divided by $\sqrt{ } 12$. The direction error is a combination of the position errors in the two planes which measure the track segment and the MCS contribution due to the material of the last (or first, in case of exit tracker) plane. For silicon tracker planes $400 \mu \mathrm{m}$ thick, $200 \mu \mathrm{m}$ pitch and $20 \mathrm{~cm}$ spaced apart, the direction error from the position measurement errors is $0.4 \mathrm{mrad}$ while the MCS contribution is already 2.4 $\mathrm{mrad}$ at $200 \mathrm{MeV}$ proton energy, increasing up to $15 \mathrm{mrad}$ at $30 \mathrm{MeV}$. In these conditions it is reasonable to neglect the former and take the MCS contribution as the direction error.

The generalization of the MLP formula and its error has been done extending the integrals of the MCS reported in [22] over the entire range of the proton path neglecting the particle energy loss in the air. The MLP uncertainty has been recalculated taken into account the errors on the entry and exit point and directions which have been propagated and added in quadrature.

The result of this generalization is shown in Fig. 1 where the following set-up, reasonable for a human head scanner, is used: $20 \mathrm{~cm}$ of water placed in between $5 \mathrm{~cm}$ of air from each side. The tracker plane is made by two sensors $320 \mu \mathrm{m}$ thick each with $200 \mu \mathrm{m}$ strip pitch.

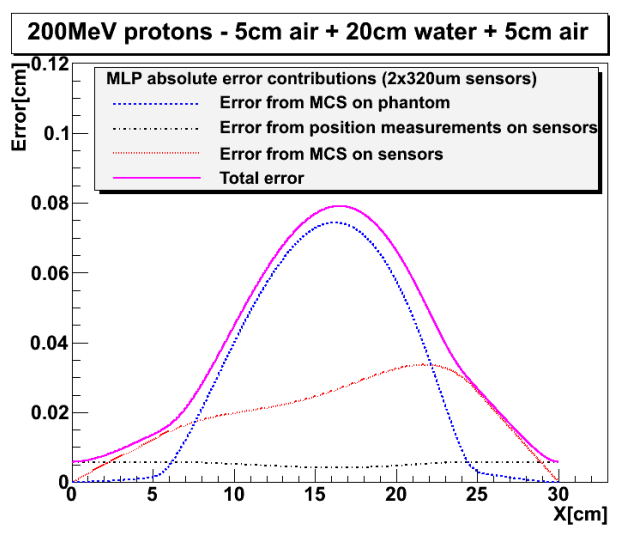

Fig. 1 Absolute error contributions to MLP from different sources. The set-up is made by: $5 \mathrm{~cm}$ air, $20 \mathrm{~cm}$ water, $5 \mathrm{~cm}$ air.

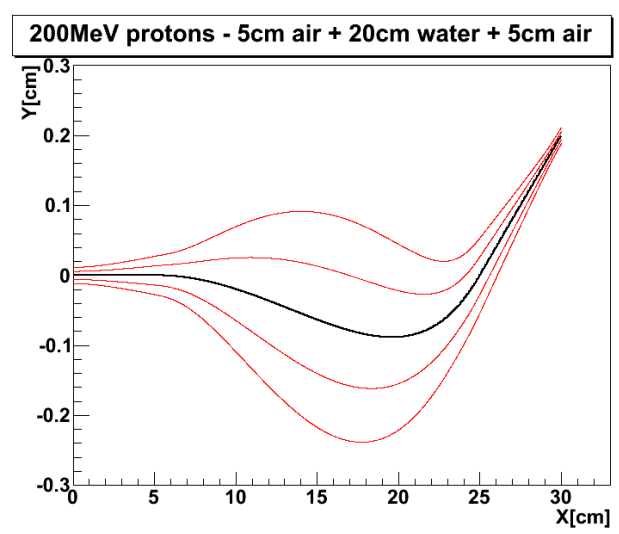

Fig. 2 MLP example for a $200 \mathrm{MeV}$ proton entering the set-up of Fig. 1 (see text); one/two sigma error envelopes are marked by the red lines.

The MLP total error in Fig. 1 (magenta, full line) mainly results from the MCS inside the phantom (blue, dashed line) except the air regions and 2-3 cm inside the phantom, where it is dominated by the MCS originating from the sensors (red, dotted line). The position measurement error on the microstrip detector (black, dashed-dotted line) is negligible except in a region of $1-2 \mathrm{~cm}$ close to the sensors. The MCS related errors are not symmetric with respect to the phantom because the proton energy loss increases these contributions as the particle penetrates the object.

Fig. 2 shows an example of an MLP of a $200 \mathrm{MeV}$ initial energy proton entering the same set-up of Fig. 1 at $y(0)=0 \mathrm{~cm}$ horizontally and exiting at $y(30)=0.2 \mathrm{~cm}$ with an angle of $40 \mathrm{mrad}$.

To allow more space between the patient and the tracker planes, the previous set-up geometry has been changed: $10 \mathrm{~cm}$ air, $20 \mathrm{~cm}$ water, $10 \mathrm{~cm}$ air. The sensor pitch and thickness are the same. As expected the larger lever arm increases the error due to the MCS in the sensors. This is shown in Fig. 3 for the absolute errors and in Fig. 4 for the relative contributions. In this more realistic configuration the MCS in the sensors is dominant also in the first and in the last 4 $\mathrm{cm}$ of the phantom. The peak value of the error is still dominated by the MCS in the phantom 
being increased with respect to the previous scenario by $50 \mu \mathrm{m}$ only. To reduce the error due to the MCS in the third plane of the tracker thinner detectors could be used in this critical position.

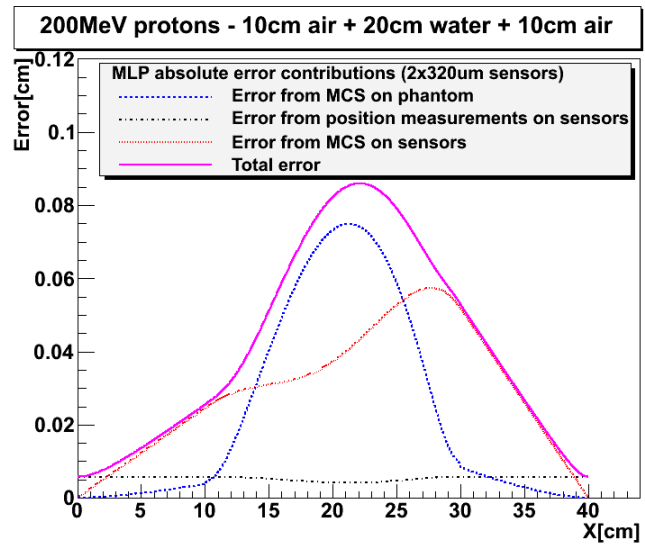

Fig. 3 Absolute error contributions to MLP from different sources. The set-up is made by: $10 \mathrm{~cm}$ air, $20 \mathrm{~cm}$ water, $10 \mathrm{~cm}$ air.

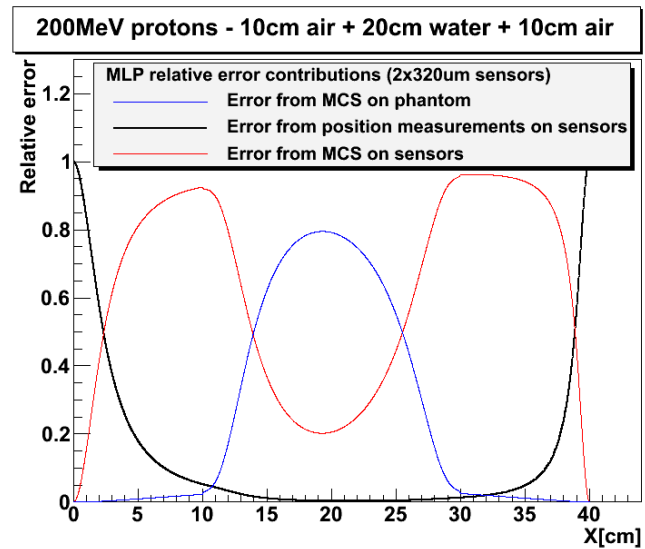

Fig. 4 Relative error contributions to MLP from different sources (same set-up as Fig. 3).

\section{Silicon Microstrip based pCT systems}

In this section the pCT systems built by the UCSC and LLUMC group and by the INFN group will be described. These apparatus, designed to demonstrate the feasibility of $\mathrm{pCT}$, are based on a Silicon Microstrip tracker and a calorimeter and are a first step towards pre-clinical systems. Larger apparatus are under construction and will be described in Sect. 6 .

The UCSC-LLUMC tracker [23] uses silicon microstrip detectors readout by ASICs both originally developed for the Gamma-Ray Large Area Space Telescope (GLAST) [24]. The single-sided, AC coupled p-on-n sensors are manufactured from high resistivity wafers of 400 $\mu \mathrm{m}$ thickness, with a strip pitch of $194 \mu \mathrm{m}$ and outer dimensions of $6.4 \times 6.4 \mathrm{~cm}^{2}$. The tracker measures hit positions with an error of about $50 \mu \mathrm{m}$ and could also be used to estimate the energy of the crossing particles in the $20-300 \mathrm{MeV}$ range. This is done measuring the specific energy loss in each silicon sensors using the time over threshold (TOT) [24-25] and then extrapolating the original particle energy. A new tracker made by four measuring planes assembled with $236 \mu \mathrm{m}$ pitch sensors, has been realized by the same collaboration and integrated with a CsI calorimeter [12]. Using this system a validation of the MLP reconstruction technique has been done measuring the proton trajectory deviation from the MLP estimation using a 'roving' tracker plane placed at different depth inside a PMMA (Polymethyl methacrylate) phantom [26].

The pCT Silicon Microstrip Tracker built by the INFN Prima collaboration [13-16] is made by four planes (Fig. 5, p1-p4) to reconstruct the particle MLP inside the target. Each plane is made by two printed circuit boards assembled back-to-back rotated by $90^{\circ}$ one respect to the other. At the center of the board a silicon microstrip sensor is glued onto a square aperture and micro bonded to eight front-end ASICs. The two sensors in a plane have theirs strips mutually perpendicular to reconstruct the track impact point on a $5.1 \times 5.1 \mathrm{~cm}^{2}$ area. The singlesided silicon microstrip sensors are of p-on-n type with a substrate $\langle 100\rangle$ orientation, a thickness 
of $200 \mu \mathrm{m}$, a strip pitch of $200 \mu \mathrm{m}$, a full depletion voltage around $75 \mathrm{~V}$ and a total number of strips of 256. The front-end ASIC consists of 32-channels, each equipped with a charge sensitive amplifier, a shaper and a comparator that produces a binary output by comparison with a threshold. The ASICs outputs are buffered and connected to a Xilinx Spartan 3 FPGA (Fig. $5 \mathrm{~d}$ ) which samples the signal lines to find active strips which are then moved into a memory.

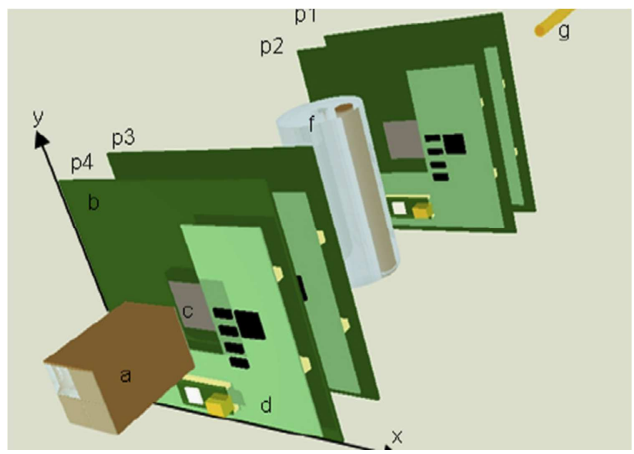

Fig. 5 pCT layout: (a) calorimeter; (b) front-end board; (c) microstrip sensor; (d) digital board; (f) phantom; (g) beam pipe; (p1-p4) tracker planes.

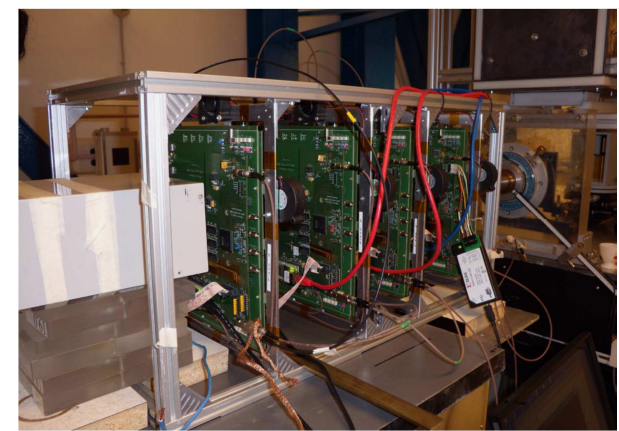

Fig. 6 The pCT prototype mounted on the INFNLNS 'Catana' beam line: the four tracker planes (green) and the calorimeter (white box) are visible

This system includes a YAG:Ce calorimeter (Fig. 5a) which is made by a $2 \times 2$ crystal matrix; each crystal has a $3 \times 3 \mathrm{~cm}^{2}$ front face and a $10 \mathrm{~cm}$ length and it is readout by a photodiode. The full system mounted on the INFN-LNS 'Catana' beam line is shown in Fig. 6.

\section{Proton tomography results}

The UCSC-LLUMC prototype described in Sect. 4 has been mounted on the LLUMC 200 $\mathrm{MeV}$ proton beam line and the radiography of a hand phantom has been reconstructed (Fig. 7) [27]. The quantity plotted in Fig. 7 as grey scale is the measured Water Equivalent Path Length (WEPL): the integral over the trajectory of the relative stopping power (RSP) of protons with respect to water. The RSP map, needed as input to the treatment planning system, is a 3D distribution reconstructed using tomographic algorithms starting from trajectory and energy information [6]. Since the RSP of bones is only about $50 \%$ higher than water, the contrast of Fig. 7 is very much reduced with respect to a common X-ray radiography. Nonetheless the RSP is the key information to set the proton beam energy to precisely reach the tumor position with the Bragg peak. Using this system an attempt to complement the WEPL measurement with the information extracted from the MCS angle inside the phantom has been pursued [27].

The INFN prototype [13-16] has been installed on the INFN-LNS (Laboratori Nazionali del Sud) $60 \mathrm{MeV}$ 'Catana' beam line to reconstruct a tomographic image of a PMMA phantom. This object has been mounted on a rotating platform inside the tracker (Fig. 8); 36 runs of about $9.5 \times 10^{5}$ events each have been taken at different angles covering the full turn [16]. For each run the energy loss profile has been extracted using the beam line as projection direction; these distributions have been used as input to a Filtered Back Projection (FBP) reconstruction algorithm [28]. The upper part of Fig.8 shows two tomographic sections of the phantom. The FBP algorithm uses a Butterworth filter [29] with cut-off at 20/128 of the Nyquist frequency $\left(f_{\mathrm{Ny}}=2.5 \mathrm{~mm}^{-1}\right.$ for a spatial sampling of $\left.200 \mu \mathrm{m}\right)$ of the projection data. In the lower part of Fig. 8 
the space resolution, calculated fitting an error function to the edge of the phantom, as function of the filter order, is shown. For a Butterworth filter of order 2 the FWHM resolution is $800 \mu \mathrm{m}$ rising to $1 \mathrm{~mm}$ if a smoother filter of order 12 is used. The density resolution is anti-correlated with the space one and changes between $6.3 \%$ and $1.4 \%$ in the same range of the filter order.

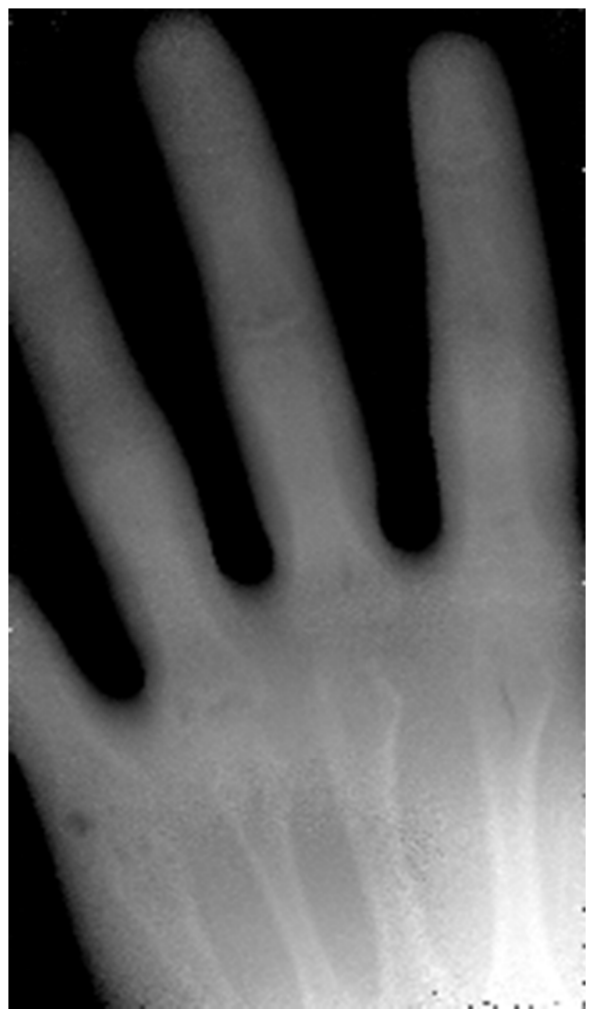

Fig. 7 WEPL radiography of a hand phantom taken with $200 \mathrm{MeV}$ energy protons.

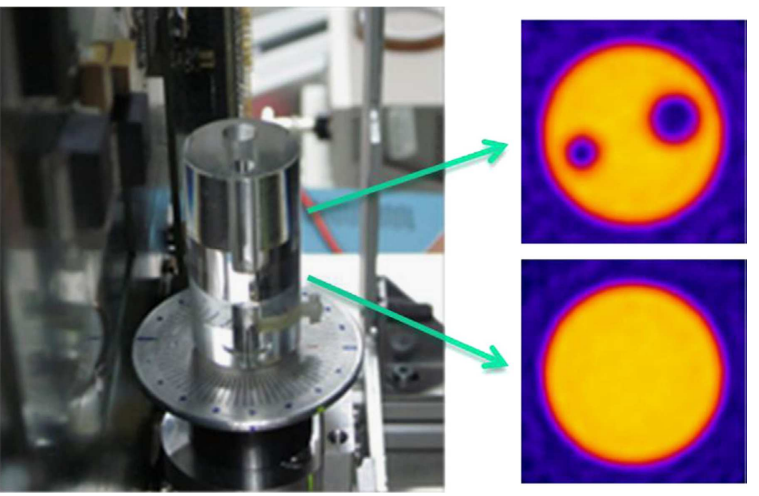

Resolution (no cut)

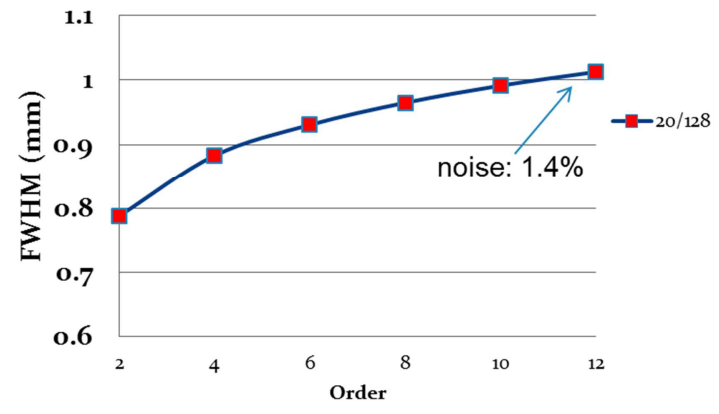

Fig. $8 \mathrm{Up}$ : phantom, mounted inside the tracker, and two sections reconstructed. Down: tomography spatial resolution as function of the FBP Butterworth filter order.

\section{Future developments}

Both groups are building similar apparatus to be used in pre-clinical tests. The major step to be achieved, aside the increase in the field of view to image a phantom of the size of a human head, is the upgrade of DAQ systems to be able to run at an average trigger rate of one $\mathrm{MHz}$ needed to reduce to a few minutes an image acquisition time. The field of view of both systems will have an aspect ratio of 4:1; images of larger objects will be reconstructed in slices.

The LLUMC/UCSC tracker (Fig. 9) will use $400 \mu \mathrm{m}$ thick $228 \mu \mathrm{m}$ pitch silicon microstrip detectors assembled in rows of four sensors each with the strips mutually orthogonal [13]; the field of view being $\sim 9 \times 36 \mathrm{~cm}^{2}$. The collaboration has performed a Geant4 Monte Carlo simulation to optimize the plane distance to minimize the RSP error for a head phantom [13]. The distance between the front and the back tracker has been found to influence the RSP sensitivity and has been set to $30 \mathrm{~cm}$ : the minimum to allow a safe rotation of a $20 \mathrm{~cm}$ diameter object. The distance between the two planes of the two tracker segments has been set to $5 \mathrm{~cm}$ as a compromise of having a compact device with an angle resolution being better than the MCS 
error contribution. The critical point limiting the performance of the apparatus is the thickness of the third plane where the lower proton energy increases the MCS contribution. The collaboration is planning to use here thinned sensors $(200 \mu \mathrm{m})$.

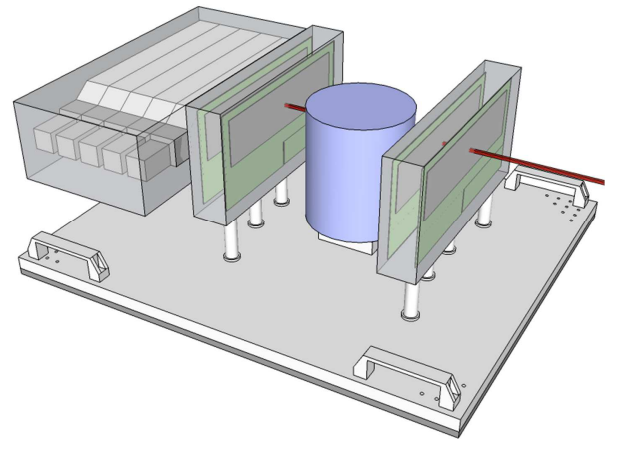

Fig. 9 Layout of the pCT head scanner under construction by the LLUMC and UCSC groups.

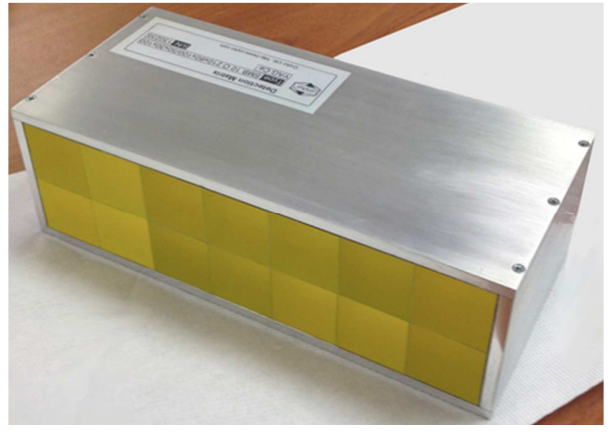

Fig. 10 The YAG:Ce crystal calorimeter of the INFN pre-clinical pCT apparatus.

To avoid dead areas within the field of view a procedure to cut the inactive edge of the sensors has been implemented [30]. The detectors are first laser scribed and cleaved then the edges are passivated using $\mathrm{SiO}_{2} / \mathrm{Si}_{3} \mathrm{~N}_{4}$ for n-type or $\mathrm{Al}_{2} \mathrm{O}_{3}$ for p-type silicon. Good performance of the 'slim edge' sensors (IV curve and charge collection efficiency) has been reported [31]. The range detector will be assembled using $4 \mathrm{~mm}$ thick Polystyrene fast scintillator blocks (Fig. 9) read-out by Silicon Photomultipliers. A range error of about $4 \mathrm{~mm} \mathrm{rms}$ has been estimated by Geant4 simulations.

The INFN groups aims at an apparatus with a field of view of $5.1 \times 20.4 \mathrm{~cm}^{2}$ [17]. Also in this case a DAQ readout frequency of $1 \mathrm{MHz}$ is foreseen to reduce the image acquisition time.

A tracker plane is realized by a single printed circuit board where the sensors, the frontend and the read out electronics are mounted. The detectors are glued on both sides of the board onto an aperture at its center: the x-measuring sensors having their strips orthogonal to the $y$ ones. Each strip of an $\mathrm{x}$ sensor is connected to a front-end channel while two strips of the $\mathrm{y}$ sensors are daisy chained onto a single channel. To reach a readout frequency of $1 \mathrm{MHz}, \mathrm{a}$ binary and parallel architecture has been implemented. Each front-end chip includes 32 channels, each of them containing a charge amplifier, a shaper and a discriminator. The thresholds are internally generated channel-by-channel by DACs which are programmed by an $\mathrm{I}^{2} \mathrm{C}$ bus. The 256 strips of a read-out group are sent to an FPGA (Xilinx Spartan 6) performing zero suppression and formatting the hit strips in an event record containing the delay with respect to the trigger time and the time-over-threshold. The FPGA, after a buffer, sends data over a differential 8-bit bus running at a speed of $1.6 \mathrm{~Gb} / \mathrm{s}$, to a 'master' FPGA which assembles the plane event and send it to a central DAQ board together with a 'global event number', produced by the calorimeter trigger processor, for event synchronization.

The calorimeter uses the same technology of the one described in Sect. 4. It is made by a $2 \times 7$ matrix of $3 \times 3 \mathrm{~cm}^{2}$ cross-section and $10 \mathrm{~cm}$ long YAG:Ce crystals (Fig. 10). Each unit has a $1.8 \times 1.8 \mathrm{~cm}^{2}$ photodiode glued on it. The 14 analogue signals are digitally converted at $5 \mathrm{MHz}$ and 14 bits. Each event contains 24 samples per crystal. Digital data is processed online to produce the trigger and, using the 8 less significant bits of the trigger counter, the event tag. 


\section{Conclusions}

The proton Computed Tomography is an imaging method potentially able to increase the effectiveness of the hadron therapy. Prototypes based on silicon microstrip trackers and scintillating calorimeters have been developed as proof of principle devices. Apparatus with a field-of-view as large as to image a human head and with a DAQ designed to run at MHz level are now under construction. This last generation of devices will provide data to develop reconstruction algorithms and to study their performance on pre-clinical phantoms completing the proof of principle of pCT. Experience gained with these systems will be useful in designing engineered devices with the final aim to be integrated with the hadron therapy systems.

\section{Acknowledgments}

The author wishes to express his gratitude to the LLUMC/UCSC and INFN collaborations for the material contained in this review. Special thanks to the organizers of Vertex 2013 for the invitation to the workshop.

\section{References}

[1] R.R. Wilson, "Radiological use of fast protons", Radiology 47 (1946) 487-491;

[2] W.H. Bragg and R. Kleeman, "On the ionization curves of radium", Philosophical Magazine, S6, (1904) 726-738;

[3] M. Jermann, PTCOG secretary, "Hadron therapy patient statistics", (March 2013), available at: http://ptcog.web.psi.ch/Archive/pat_statistics/Patientstatistics-updateMar2013.pdf;

[4] U. Schneider et al., "The calibration of CT Hounsfield units for radiotherapy treatment planning", Phys. Med. Biol., 41 (1996) 111-124;

[5] B. Schafner and E. Pedroni, "The precision of proton range calculations in proton radiotherapy treatment planning: experimental verification of the relation between CT-HU and proton stopping power", Phys. Med. Biol., 43 (1998) 1579-1592;

[6] T. Li et al., "Reconstruction for proton computed tomography by tracing proton trajectories: A Monte Carlo study", Med. Phys., 33 (3) (2006) 699-706;

[7] M. H. Phillips et al., "Stereotactic radiosurgery: A review and comparison of methods", J. Clin. Oncol. 12-5, (1994) 1085-1099;

[8] A.M. Koehler, "Proton radiography", Science 160 (1968) 303-304;

[9] U. Schneider and E. Pedroni, "Proton radiography as a tool for quality control in proton therapy", Med. Phys., 22 (1995) 353-363;

[10] P. Pemler et al., "A detector system for proton radiography on the gantry of the Paul-ScherrerInstitute”, Nucl. Instr. and Meth. A 432 (1999) 483-495;

[11] L.R. Johnson et al., "Initial studies on proton computed tomography using a silicon strip detector telescope", Nucl. Instr. and Meth. A 514 (2003) 215-223;

[12] R. Schulte et al., "Conceptual design of a proton computed tomography system for applications in proton radiation therapy", IEEE Trans. Nucl. Sci., 51-3 (2004) 866-872; 
[13] H.F.W. Sadrozinski et al., "Development of a head scanner for proton CT”, Nucl. Instr. and Meth. A 699 (2013) 205-210;

[14] G. Cirrone et al. "The Italian project for a proton imaging device”, Nucl. Instr. and Meth. A 576 (2007) 194-197;

[15] M. Scaringella et al.," The PRIMA (Proton Imaging) collaboration: development of a proton Computed Tomography apparatus”, Nucl. Instr. and Meth. A 730 (2013) 178-183;

[16] E. Vanzi et al., "The PRIMA collaboration: preliminary results in FBP reconstruction of $p C T$ data”, Nucl. Instr. and Meth. A 730 (2013) 184-190;

[17] C. Civinini et al., "Recent results on the development of a proton computed Tomography system”, Nucl. Instr. and Meth. A 732 (2013) 573-576;

[18]Y. Saraya et al., "Study of spatial resolution of proton computed tomography using a silicon strip detector", Nucl. Instr. and Meth. A 735 (2013) 485-489;

[19] U. Amaldi et al., "Construction, test and operation of a proton range radiography system”, Nucl. Instr. and Meth. A 629 (2011) 337-344;

[20] S. Braccini, et al., "First results on proton radiography with nuclear emulsion detectors", JINST, 5 (2010) P09001;

[21] D.C. Williams, "The most likely path of an energetic charged particle through a uniform medium”, Phys. Med. Biol., 49 (2004) 2899;

[22] R.W. Shulte et al., "A maximum likelihood proton path formalism for application in proton computed tomography”, Med. Phys., 35 (11) (2008) 4849-4856;

[23] B. Keeney et al., "A silicon telescope for applications in nanodosimetry”, IEEE Trans. Nucl. Sci., NS-49 (2002) 1724;

[24] E. do Couto e Silva et al., "Results from the beam test of the engineering model of the GLAST large area telescope”, Nucl. Instr. and Meth. A 474 (1) (2001)19-37;

[25] R.P. Johnson et al., “An Amplifier-Discriminator Chip for the GLAST Silicon-Strip Tracker”, IEEE Trans. Nucl. Sci., NS-45 (1998) 927;

[26] M. Bruzzi et al., "Prototype Tracking Studies for Proton CT", IEEE Trans. Nucl. Sci., 54-1 (2007) 140-145;

[27] T. Paultz et al., "200 MeV Proton Radiography Studies with a Hand Phantom Using a Prototype Proton CT Scanner”, in: Proceedings of the 2012 IEEE NSS-MIC (2012);

[28] A.C. Kak and M. Slaney, "Principles of Computerized Tomographic Imaging”, IEEE Press, 1988, 〈http://www.slaney.org/pct/index.html〉;

[29] S. Butterworth, "On the theory of filter amplifiers", Experimental Wireless and the Wireless Engineer, 7 (1930) 536-541;

[30] M. Christophersen et al., "Alumina and silicon oxide/nitride sidewall passivation for Pand N-type Sensors”, Nucl. Instr. and Meth. A 699 (2013)14-17;

[31] R. Mori et al., "Charge collection measurements on slim-edge microstrip detectors", JINST 7 (2012) P05002. 